\title{
Validation of the 5-D Itch Scale in Three Ethnic Groups and Exploring Optimal Cutoff Values Using the Itch Numerical Rating Scale
}

\author{
H. N. Cheung $\mathbb{D}^{1}{ }^{1}$ Y. S. Chan, ${ }^{2}$ and N. H. Hsiung $\mathbb{D}^{3}$ \\ ${ }^{1}$ Department of Social Science, Hong Kong Metropolitan University, Hong Kong \\ ${ }^{2}$ Department of Applied Social Studies, Hong Kong Polytechnic University, Hong Kong \\ ${ }^{3}$ Department of Nursing, Asia University, Taiwan \\ Correspondence should be addressed to H. N. Cheung; cheunghn@ouhk.edu.hk
}

Received 19 May 2021; Revised 15 November 2021; Accepted 29 November 2021; Published 13 December 2021

Academic Editor: Adam Reich

Copyright (c) $2021 \mathrm{H}$. N. Cheung et al. This is an open access article distributed under the Creative Commons Attribution License, which permits unrestricted use, distribution, and reproduction in any medium, provided the original work is properly cited.

\begin{abstract}
Background. Chronic pruritus is a common and distressing condition that has serious emotional and psychosocial consequences. Due to its subjective nature, self-report questionnaires are widely implemented as cost-effective measures to gauge the severity of chronic pruritus. The current study is aimed at validating the 5-D itch scale in three ethnic groups-Black, Asian, and Hispanic-with the well-validated Itch Numerical Rating Scale (NRS) and Worst Itch NRS (WI-NRS) and developing its cutoff value using receiver operating characteristics (ROC) and inspection of the area under the curve (AUC) across ethnic groups. At the same time, it is aimed at comparing the concurrent prevalence of itch and depression in these populations, who often form ethnic minorities in many countries. The current study addresses the knowledge gap of cultural adaptation of the 5-D pruritus scale for greater usage. Methods. Community samples of three ethnic groups were recruited from an online platform of Qualtrics and administered the self-report questionnaires of Itch-NRS, 5-D itch scale, and Patient Health Questionnaire-9 (PHQ-9) to measure their pruritus domains, itch intensity, depression screening, and its severity. Informed consent was obtained from all participants. Subgroup analysis was conducted, including concurrent validity and cutoff values compared between each ethnic group. Concurrent prevalence of itch and depression was evaluated using the cutoff value of Itch-NRS and PHQ-9. Result. A total of 2323 participants were included in the study. A significant positive correlation $(p<0.001)$ was found between the Itch-NRS, WI-NRS, and 5-D itch scale. The cutoff value of the 5-D itch scale was established for the three ethnic groups using ROC, with a cutoff value of Itch-NRS as a reference. Conclusions. The 5-D itch scale has demonstrated sound psychometric properties in three ethnic groups and is closely related to Itch-NRS. The analysis of the cutoff value of the 5-D itch scale suggests that different cutoff values should be considered to reduce the inflation of pruritus severity.
\end{abstract}

\section{Introduction}

Pruritus (or itch) has been an emerging but important topic in the area of dermatology that impacts the quality of life. It is identified as one of the most prevalent skin condition [1], signified by unpleasant sensations of burning and tingling. Itch is often followed by the urge to scratch as a reflex-like response [2], which leads to the itch-scratch cycle of reliving an intolerable itch [3] by scratching [4]. However, the itchscratch cycle precedes the worsening of itch due to its serotonin-releasing nature for reward and further worsens lesion which sustains itch [5].

Approximately one-third of the population experiences itching weekly. When pruritus lasts over 6 weeks, it becomes chronic and thus is more difficult to treat [6]. Over 20 million Americans are reported to experience chronic pruritus [7]. A recent epidemiological study in Germany $(n=334)$ recorded over a third of patients experiencing pruritus, close to $90 \%$ of whom had chronic pruritus [8]. An equally high prevalence of $40 \%$ was documented in Japan [9] and a 
comparable $38 \%$ in a Polish study [10]. However, the prevalence has been scarcely reported in Asian populations. Many studies have focused on investigating it as a comorbid condition in outpatients with eczema and dermatitis [11] and pruritus in older populations [12]. Chronic pruritus imposes a large burden on patients' quality of life, comparable to that of chronic pain [13]. Patients with chronic pruritus have been found to have worsened health-related quality of life in both genders [14], particularly affecting daily activities, sleep patterns, and interpersonal relationships. It is also associated with depression [15] and other psychological disorders, such as anxiety and sleep disorders, due to the irritating feeling of itch and visible skin lesions. This results in a vicious cycle in which psychological stress may further exacerbate pruritus or pain [16] and the frequency of scratching. Psychiatric comorbidities such as parasitosis, hallucinations, and compulsive scratching syndromes and increased life stresses [17] are also prevalent in pruritus patients, and suicidal ideation is a commonly reported consequence. A large-scale cross-sectional study in 13 European countries $(N=3530)$ reported $14.1 \%$ depression prevalence among dermatological patients with pruritus [18].

Chronic pruritus relies on self-report assessments due to its subjective nature. The unidimensional Itch Numerical Rating Scale (Itch-NRS) has been developed as a patientreported outcome to provide cost-efficient screening tools for measuring itch severity. Itch-NRS measures itch severity in the past 24 hours. It is also one of the most popular scales used in clinical settings and is commonly used as a reference to validate other pruritus scales. Itch-NRS has been validated in several languages with good psychometric properties (e.g., $[19,20]$. It has also been further developed to become a oneitem Worst Itch NRS (WI-NRS) that measures the peak itch severity in the past 24 hours in patients with atopic dermatitis [20]. Both had psychometric properties widely validated across populations, including reliability and validity $[20,21]$. The scores of Itch-NRS range from no (0 points), mild (1-3 points), moderate (4-6 points), severe (7-8 points), to very severe ( $\geq 9$ points) [22]. A clinical study on patients with chronic pruritus supported a score range of NRS to be 3-79, 4-6-9, and 4-7-9 with good correlation coefficients between NRS and VAS [23]. The cutoff value of Itch-NRS indicates a moderate level of symptoms is considered clinically symptomatic, while a cutoff value was yet to be developed for the WI-NRS. However, the NRS only provides an overall measure of severity while lacking a multidimensional evaluation of itch. To overcome this shortcoming, another popular self-report scale, the 5-D itch scale [24], a multidimensional measure of itch duration, degree, direction, disability, and distribution, has been validated extensively across clinical groups, such as patients with burns, psoriasis, and haemodialysis $[22,25,26]$, with impressive concurrent validity. Despite its well-researched reliability and validity in various languages such as Spanish [27], Chinese [28], and Urdu [29], the cutoff value of the 5-D itch scale has not been fully explored among healthy community adults in various ethnic groups for screening purposes. Considering the high prevalence of pruritus on its own in general populations, a brief and valid scale that can also screen adults with and without pruritus could have potentially important clinical implications, especially in primary care settings. In addition, previous validation studies of the 5-D itch scale focused heavily on its validity and reliability. Hence, the cross-cultural comparison association between itch and depression in ethnic groups other than Caucasian, such as Asian, Black/African, and Hispanic, should be explored. The current study is aimed at validating the 5-D itch scale against Itch-NRS and WI-NRS on the concurrent validity and developing a cutoff value of the 5-D itch scale based on the reference of the Itch-NRS cutoff value. It is also aimed at providing further insight into the prevalence of itch and its possible cooccurring condition such as depression among the three ethnic groups using Itch-NRS and the Patient Health Questionnaire-9 (PHQ-9). The current study provides important exploratory results in the psychometric properties of the 5-D itch scale across ethnic groups as well as the prevalence of itch and other conditions across ethnic groups.

\section{Materials and Methods}

2.1. Participants and Procedures. A community sample of 2323 individuals across three ethnic groups worldwide, Asian (Chinese, Japanese, Korean, Indian, and other Asian ethnicity), Black, and Hispanic, was collected through an online platform by Qualtrics. Data collection lasted from 11 January to 12 February 2021. Potentially eligible participants above 18 who fit the three specified ethnic groups and could read English were contacted through email with an information sheet and consent form for their consideration. After providing written consent, participants were given a brief questionnaire to collect information on demographics, including age, gender, current skin diseases, and presence of chronic pruritus; a brief one-item questionnaire of the Itch-NRS; WI-NRS; and a 5-item 5-D itch scale. On the questionnaire, they were also assessed on their mental health state using depression scales as the PHQ-9.

\subsection{Measures}

2.2.1. The Itch Numerical Rating Scale (NRS). The Itch-NRS is a self-reported, brief, unidimensional itch intensity scale facilitating a Likert scale from 0 (no itch) to 10 (worst imaginable itch) for increasing self-perception of itch severity. With regard to pruritus, two separate scales are used. The Itch-NRS measures the itch on average within the past 24 hours, and the Worst Itch NRS (WI-NRS) scale measures the worst itch in the past 24 hours. It has been validated to have good psychometric properties, including a high significant concurrent validity with visual analogue scale (VAS) $(r>0.8 ; p<0.01)$ and a high test-retest reliability with intraclass correlation coefficient of 0.8 [19]. In the current study, a Pearson correlation of $0.87(p<0.001)$ was found between Itch-NRS and WI-NRS.

2.2.2. The 5-D Itch Scale. The assessment of prevalence was conducted using the 5-D itch scale developed by Elman et al. [24], a brief self-report questionnaire including five domains of pruritus: duration, degree, direction, disability, 
and direction. The Chinese version has been translated into traditional Chinese characters [22] with good psychometric properties: Cronbach's alpha of 0.734 , strong and significant correlation with a visual analogue scale, and sensitivity to change over time. Cronbach's alpha for the current sample was 0.93 .

2.2.3. Patient Health Questionnaire. The PHQ-9 is a widely used depression screening tool in primary care settings [30]. The nine-item self-report scale is documented to have acceptable concurrent validity. In differentiating depressed from nondepressed individuals, a cutoff value of 10 yielded high sensitivity (0.88) and specificity $(0.85)$ [31, 32].

2.3. Data Analysis. SPSS 16 (IBM, 2020) was used for subgroup analysis. Chi-square tests of independence were performed to compare the differences between ethnic groups in demographic variables such as gender, mental health history, skin conditions, presence of itch by Itch-NRS cutoff of 4 , presence of depression by PHQ-9, and presence of itch above 6 weeks. The significance level was set at $p<0.05$.

Regarding the psychometric properties of the 5-D itch scale, the internal consistency of the 5-D itch scale was indicated by Cronbach's alpha. The 5-D itch scale was also compared with the Itch-NRS and WI-NRS for concurrent validity by Spearman correlation. The cutoff value of the 5-D itch scale was established by receiver operating characteristics (ROC) curve across three ethnic groups by comparing with the cutoff value of Itch-NRS as reference. Cutoff value of 4 of the Itch-NRS was tested against the 5-D itch scale, as it indicates moderate severity of pruritus and beyond this value would be clinically regarded as symptomatic pruritus [22]. The cutoff value was chosen because it was supported in validation of Itch-NRS in patients with other skin disease such as haemodialysis [22]. In addition, although Reich et al. [23] proposed the best cutoff value of 3 in ItchNRS, a cutoff value of 4 was also supported in their study. ROC curves are indicators of combined sensitivity and specify all possible cutoff points with the area under the curve (AUC) measuring diagnostic accuracy. An AUC of $\geq 0.9$ is regarded as "excellent," 0.8-0.9 considered "good," and 0.7-0.8 is considered "fair" [33]. The AUCs of the Itch-NRS and the 5-D itch scale were compared in the study. As the Itch-NRS was the sole diagnostic instrument, an optimal cutoff value was selected for the best trade-off between sensitivity and specificity.

\section{Results}

A total of 2323 questionnaires were completed at the viable platform for data collection. The descriptive statistics of participants from the three ethnic groups are listed in Table 1. Participants reported a mean age of 35.17 (standard deviation $(\mathrm{SD})=14.26)$. The population included $46.58 \%$ Asian, 27.38\% Black/African, and 26.04\% Hispanic. The majority of participants were female $(65.5 \%)$. The study population comprised 339 participants (14.59\%) with known mental disorders during the test period, including depression
(39.23\%), anxiety (21.82\%), bipolar disorder $(7.67 \%)$, and stress-related disorders (20.05\%).

The chi-square test of independence of the differences between ethnic groups in demographic variables showed that there was a significant difference of participants with history of mental disorders among ethnic groups. A lower percentage $(11.8 \%)$ of Black participants showed mental disorders when compared to Asian participants $(12.4 \%)$ and Hispanic participants $(21.5 \%)\left(\chi^{2}(2, N=2323)=31.31\right.$, $p<0.001)$. Regarding skin conditions, $16.9 \%$ of Asian participants perceived a presence of adverse skin condition. Among them, over half reported eczema (51.9\%). A similar percentage of $13.2 \%$ and $14.9 \%$ of Black and Hispanic participants, respectively, reported the presence of skin condition, while eczema was also the most common condition, accounting for around a third prevalence $\left(\chi^{2}(18, N=2323)=48.06\right.$, $p<0.001)$.

To test the occurrence of pruritus and depression, the prevalence of itch and depression was indicated by the percentage of the sample who scored above the cutoff of Itch-NRS and PHQ-9, respectively, based on the cutoff values of NRS $\geq 4$ and PHQ $\geq 10$ [34]. A significant effect was found in the ethnic difference of itch by $\mathrm{NRS} \geq 4 \quad\left(\chi^{2}(2, N=2323)=26.1\right.$, $p<0.001)$ and concurrent itch and depression. The Black group reported the highest percentage of $23.4 \%$, followed by the Hispanic group (21.3\%) and the Asian group (12.8\%) $\left(\chi^{2}(3, N=2323)=37.53, p<0.001\right)($ Table 1$)$.

3.1. Convergent Validity. Subgroup analysis of ethnic groups suggested a significant positive correlation $(p<0.001)$ from Spearman correlation between the one-item Itch-NRS, WI-NRS, and 5-D itch scale (Table 2). The result suggested good convergent validity and thus high agreement of both the Itch-NRS and WI-NRS with the 5-D itch scale regarding the measurement of general and peak pruritus using the 5-D itch scale.

3.2. Cutoff Value of the 5-D Itch Scale by ROC. On the basis of the NRS cutoff value of 4 , the ROC curve for the Asian sample is shown in Figure 1. The AUC was 0.87 (95\% CI: $0.84-0.90)$. A cutoff value of 8.5 yielded a sensitivity of $80.5 \%$ and 1 -specificity of $80.3 \%$.

The ROC curve for the Black/African sample is shown in Figure 2. The AUC was 0.86 (95\% CI: 0.83-0.89). A cutoff value of 8.5 yielded a sensitivity of $81.4 \%$ and 1 -specificity of $77.8 \%$.

The ROC curve for the Hispanic sample is shown in Figure 3. The AUC was 0.86 (95\% CI: 0.84-0.90). A cutoff value of 9.5 yielded a sensitivity of $79.7 \%$ and 1 -specificity of $83.0 \%$.

\section{Discussion}

This study is aimed at exploring the psychometric properties of the 5-D itch scale and its cutoff value across community Asian, Black, and Hispanic samples using the cutoff value of Itch-NRS as a reference scale. It is also aimed at comparing the occurrence of itch and depression using the 
TABle 1: Descriptive statistics of three ethnic groups.

\begin{tabular}{|c|c|c|c|c|}
\hline Variables & Asian $(n=1082)$ & Black/African $(n=636)$ & Hispanic $(n=605)$ & $\chi^{2}$ \\
\hline $\begin{array}{l}\text { Age } \\
\text { Mean (SD) }\end{array}$ & $37.0(14.74)$ & $34.0(14.5)$ & $33.1(12.7)$ & - \\
\hline \multicolumn{5}{|l|}{$\begin{array}{l}\text { Gender } \\
n(\%)\end{array}$} \\
\hline Female & $700(64.7)$ & $422(66.4)$ & $400(66.1)$ & \multirow[t]{2}{*}{0.62} \\
\hline Male & $382(35.3)$ & $214(33.6)$ & $205(33.9)$ & \\
\hline \multicolumn{5}{|l|}{$\begin{array}{l}\text { History of mental disorders } \\
n(\%)\end{array}$} \\
\hline Yes & $134(12.4)$ & $75(11.8)$ & $130(21.5)$ & \multirow[t]{2}{*}{$31.31^{* *}$} \\
\hline No & $948(87.6)$ & $561(88.2)$ & $475(78.5)$ & \\
\hline \multicolumn{5}{|l|}{$\begin{array}{l}\text { Presence of skin diseases } \\
n(\%)\end{array}$} \\
\hline Yes & $183(16.9)$ & $84(13.2)$ & $90(14.9)$ & \multirow[t]{2}{*}{4.31} \\
\hline No & $899(51.3)$ & $552(86.8)$ & $515(85.1)$ & \\
\hline \multicolumn{5}{|l|}{$\begin{array}{l}\text { Current skin diseases } \\
n(\%)\end{array}$} \\
\hline Acne & $14(7.6)$ & $3(3.6)$ & $10(11.1)$ & \multirow[t]{9}{*}{$48.06^{* *}$} \\
\hline Atopic dermatitis & $95(51.9)$ & $24(28.6)$ & $30(33.3)$ & \\
\hline Dryness & $17(9.3)$ & $6(7.1)$ & $11(12.2)$ & \\
\hline Psoriasis & $4(2.2)$ & $2(2.4)$ & $5(5.6)$ & \\
\hline HS & $1(0.5)$ & $1(1.2)$ & $1(1.1)$ & \\
\hline Hives & $3(1.6)$ & $1(1.2)$ & $0(0.0)$ & \\
\hline Rashes and fungus infection & $12(6.6)$ & $2(2.4)$ & $2(2.2)$ & \\
\hline Lupus & $0(0.0)$ & $2(2.4)$ & $0(0.0)$ & \\
\hline Others and unspecified & $37(20.2)$ & $43(51.2)$ & $31(34.4)$ & \\
\hline \multicolumn{5}{|c|}{$\begin{array}{l}\text { Presence of depression PHQ-9 } \geq 10 \\
n(\%)\end{array}$} \\
\hline Yes & $334(30.9)$ & $287(45.1)$ & $309(51.1)$ & \multirow[t]{2}{*}{$75.45^{* *}$} \\
\hline No & $748(69.1)$ & $349(54.9)$ & $296(48.9)$ & \\
\hline \multicolumn{5}{|l|}{$\begin{array}{l}\text { Presence of pruritus Itch-NRS } \geq 4 \\
n(\%)\end{array}$} \\
\hline Yes & $261(24.1)$ & $226(35.5)$ & $182(30.1)$ & \multirow[t]{2}{*}{$26.10^{* *}$} \\
\hline No & $821(75.9)$ & $410(64.5)$ & $423(69.9)$ & \\
\hline \multicolumn{5}{|c|}{$\begin{array}{l}\text { Presence of both depression and pruritus } \\
n(\%)\end{array}$} \\
\hline Yes & $138(12.8)$ & $149(23.4)$ & $129(21.3)$ & \multirow[t]{2}{*}{$37.53^{* *}$} \\
\hline No & $944(87.2)$ & $487(76.6)$ & $476(78.7)$ & \\
\hline \multicolumn{5}{|c|}{$\begin{array}{l}\text { Presence of itch symptoms over } 6 \text { weeks } \\
n(\%)\end{array}$} \\
\hline Yes & $196(18.1)$ & $107(16.8)$ & $94(15.5)$ & \multirow[t]{2}{*}{1.86} \\
\hline No & 886 (81.9) & $529(83.2)$ & $511(84.5)$ & \\
\hline
\end{tabular}

${ }^{* *} p<0.001$.

cutoff value of Itch-NRS and PHQ-9 across the three ethnic groups.

The demographic characteristics of the samples reported a significant variation in self-report of itch, depression, and adverse skin conditions. In coherent with the COVID situation of mental health, the prevalence of depression increased to a third of the sample in the Asian group and over half in the Hispanic group. The result is consistent with the finding that Hispanic adults experienced a greater level of psychosocial stress due to insufficient food and stable living conditions than adults in other ethnic groups [35]. Subsequently, the worsening in mental health could fuel the itch-psyche association as reflected in the cooccurring depression itch condition. It aligns with the positive association between chronic pruritus and depression [36]. Using the cutoff value of the Itch-NRS $\geq 4$ and PHQ-9 $\geq 10$, the Black/African 
TABLE 2: Spearman correlation between Itch-NRS and WI-NRS with 5-D itch scale.

\begin{tabular}{lcc}
\hline & Itch-NRS/5-D & WI-NRS/5-D \\
\hline Asian & $0.67^{*}$ & $0.69^{*}$ \\
Black/African & $0.64^{*}$ & $0.63^{*}$ \\
Hispanic & $0.67^{*}$ & $0.68^{*}$ \\
\hline
\end{tabular}

$p<0.001$.

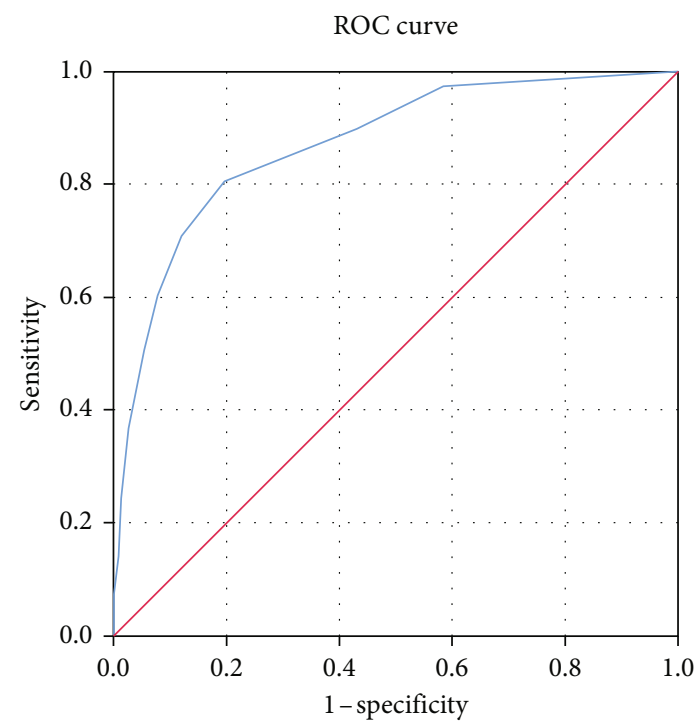

Diagonal segments are produced by ties.

FIgURE 1: ROC curve and AUC for Asian sample.

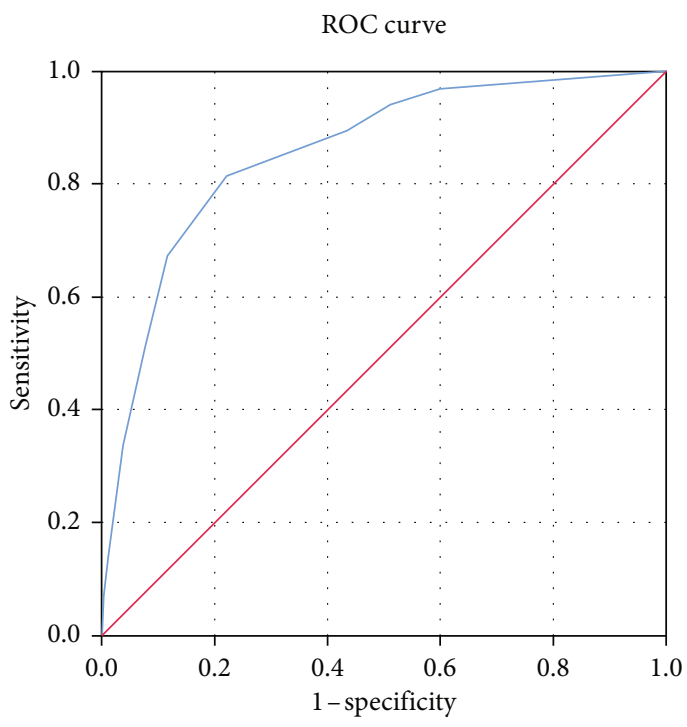

Diagonal segments are produced by ties.

FIgURE 2: ROC curve and AUC for Black/African sample.

group reported the highest percentage of itch and concurrent itch and depression, followed by the Hispanic group. This finding is coherent with that reported by Ruprecht et al. [37] that Black and Latin people are more vulnerable

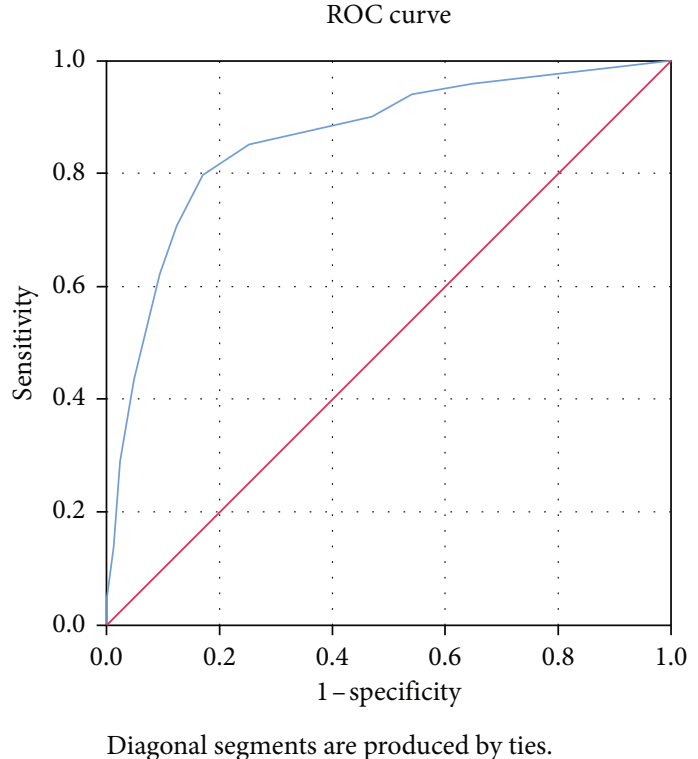

Figure 3: ROC curve and AUC for Hispanic sample.

to mental distress, possibly due to being in a more disadvantaged economic position. At the same time, they are also at a higher risk of physical illnesses such as diabetes and unsuppressed HIV viral load, which could also contribute to selfreported pruritus [38]. The current study thus highlights the extra care in mental and itch areas required for ethnic minorities, especially for Black and Hispanic people across the world in dermatology and psychology.

The results of the current study reported good psychometric properties in a community sample of three ethnic groups, as indicated by the significantly high concurrent validity of the 5-D itch scale with the Itch-NRS and WINRS. The findings show that the multidimensional 5-D itch scale is a useful screening tool to reflect the severity of itch among the three ethnic populations. Using NRS $\geq 4$ as a reference, the cutoff value of the 5-D itch scale identified the same cutoff value for the Asian and Black/African groups (8.5) but a higher cutoff value for the Hispanic group (9.5), yielding similar high sensitivity and specificity $(\sim 0.8)$. This highlights the importance of cultural adaptation of cutoff values of the 5-D itch scale to increase the accuracy of classifying individuals with and without itch. In particular, Hispanic ethnic groups may report a more elevated itch score and using the same cutoff value as for other ethnic groups would result in an inflation of itch identification. In addition, the cutoff values of the 5-D itch scale across ethnic groups in the current study are lower than the score of reported moderate pruritus (i.e., 12) in previous studies [22]. A more conservative cutoff value might be an advantage in community screening to lower the chance of false negative.

The current study addresses the important gap in the literature in cross-cultural validation of the 5-D itch scale, as our results indicate that a cutoff value is usually not established or validated across ethnic groups. It also opens up the opportunity for further investigation into the cutoff scores across cultures in comparison to the clinical 
population. It has important implication in developing a valid screening tool for pruritus in community sample potentially in primary care settings.

There are some potential limitations of this study. Recruiting samples from a crowdsourcing Qualtrics platform has been reported to be skewed towards a more elevated score of mental health than the general population. In the current study, the demographics of the sample showed slight skewness towards males. A clinical sample is needed as a comparison group to establish the cutoff value of the 5-D itch scale in the future. Finally, an elevated score of the PHQ-9 resulting from COVID-19 might influence the result of the concurrent prevalence of individuals with depression and itch.

\section{Conclusion}

In the validation studies, the 5-D itch scale was valid and reliable among different ethnic groups. A cutoff score with high sensitivity and specificity has been established in the 5-D itch scale using the Itch-NRS as a reference. The study is also coherent with previous literature that Black and Hispanic people are more vulnerable to concurrent itch and depression than Asians.

\section{Data Availability}

The data is available upon request. Please contact the corresponding author at cheunghn@hkmu.edu.hk.

\section{Conflicts of Interest}

The authors declare that there is no conflict of interest regarding the publication of this paper.

\section{Acknowledgments}

The work described in this paper was substantially supported by the Katie Shu Pui Charitable Trust (KS 2020/30) and the Public and Social Policy Research Centre, which had been established and supported by a grant from the Research Grants Council of the Hong Kong Special Administrative Region, China (UGC/IDS16/18).

\section{References}

[1] E. Weisshaar and F. Dalgard, "Epidemiology of itch: adding to the burden of skin morbidity," Acta Dermato-Venereologica, vol. 89, no. 4, pp. 339-350, 2009.

[2] G. Rinaldi, "The itch-scratch cycle: a review of the mechanisms," Dermatology Practical Conceptual, vol. 9, no. 2, pp. 90-97, 2019.

[3] S. G. Leknes, S. Bantick, C. M. Willis, J. D. Wilkinson, R. G. Wise, and I. Tracey, "Itch and motivation to scratch: an investigation of the central and peripheral correlates of allergenand histamine-induced itch in humans," Journal of Neurophysiology, vol. 97, no. 1, pp. 415-422, 2007.

[4] Z. Q. Zhao, X. Y. Liu, J. Jeffry et al., "Descending control of itch transmission by the serotonergic system via 5-HT1A- facilitated GRP-GRPR signaling," Neuron, vol. 84, no. 4, pp. 821-834, 2014.

[5] H. Murota and I. Katayama, "Exacerbating factors of itch in atopic dermatitis," Allergology International, vol. 66, no. 1, pp. 8-13, 2017.

[6] S. Stander, E. Weisshaar, T. Mettang et al., "Clinical classification of itch: a position paper of the International Forum for the Study of Itch," Acta Dermato-Venereologica, vol. 87, no. 4, pp. 291-294, 2007.

[7] N. K. Mollanazar, S. D. Koch, and G. Yosipovitch, "Epidemiology of chronic pruritus: where have we been and where are we going?", Current Dermatology Reports, vol. 4, no. 1, pp. 20-29, 2015.

[8] M. E. Kopyciok, H. F. Stander, N. Osada, S. Steinke, and S. Stander, "Prevalence and characteristics of pruritus: a oneweek cross-sectional study in a German dermatology practice," Acta Dermato-Venereologica, vol. 96, no. 1, pp. 50-55, 2016.

[9] S. Oeda, H. Takahashi, H. Yoshida et al., "Prevalence of pruritus in patients with chronic liver disease: a multicenter study," Hepatology Research, vol. 48, no. 3, pp. E252-E262, 2018.

[10] J. Szczech, A. Wiatrowski, L. Hirnle, and A. Reich, "Prevalence and relevance of pruritus in pregnancy," BioMed Research International, vol. 2017, 6 pages, 2017.

[11] X. Wang, L. Li, X. Shi, P. Zhou, and Y. Shen, "Itching and its related factors in subtypes of eczema: a cross-sectional multicenter study in tertiary hospitals of China," Scientific Reports, vol. 8, no. 1, pp. 10754-10757, 2018.

[12] J.-N. Lin, D.-L. Chiang, and T.-Y. Chen, "A psychometric evaluation of the Chinese version of the 5D itch scale in Taiwanese elderly," International Journal of Applied Science and Technology, vol. 9, no. 1, pp. 1-9, 2019.

[13] S. P. Kini, L. K. DeLong, E. Veledar, A. M. McKenzie-Brown, M. Schaufele, and S. C. Chen, "The impact of pruritus on quality of life: the skin equivalent of pain," Archives of Dermatology, vol. 147, no. 10, pp. 1153-1156, 2011.

[14] B. Warlich, F. Fritz, N. Osada et al., "Health-related quality of life in chronic pruritus: an analysis related to disease etiology, clinical skin conditions and itch intensity," Dermatology, vol. 231, no. 3, pp. 253-259, 2015.

[15] A. Reich, E. Hrehorow, and J. C. Szepietowski, "Pruritus is an important factor negatively influencing the well-being of psoriatic patients," Acta Dermato-Venereologica, vol. 90, no. 3, pp. 257-263, 2010.

[16] U. Gieler, V. Niemeier, B. Brosig, and J. Kupfer, "Psychosomatic aspects of pruritus," Dermatology and Psychosomatics, vol. 3, no. 1, pp. 6-13, 2002.

[17] C. Schut, S. Bosbach, U. Gieler, and J. Kupfer, "Personality traits, depression and itch in patients with atopic dermatitis in an experimental setting: a regression analysis," Acta Dermato-Venereologica, vol. 94, no. 1, pp. 20-25, 2014.

[18] F. J. Dalgard, A.. Svensson, J. A. Halvorsen et al., "Itch and Mental Health in Dermatological Patients across Europe: A Cross- Sectional Study in 13 Countries," Journal of Investigative Dermatology, vol. 140, no. 3, pp. 568-573, 2020.

[19] N. Q. Phan, C. Blome, F. Fritz et al., "Assessment of pruritus intensity: prospective study on validity and reliability of the visual analogue scale, numerical rating scale and verbal rating scale in 471 patients with chronic pruritus," Acta DermatoVenereologica, vol. 92, no. 5, pp. 502-507, 2012.

[20] G. Yosipovitch, M. Reaney, V. Mastey et al., "Peak Pruritus Numerical Rating Scale: psychometric validation and responder definition for assessing itch in moderate-to-severe 
atopic dermatitis," British Journal of Dermatology, vol. 181, no. 4, pp. 761-769, 2019.

[21] A. Reich, C. Riepe, Z. Anastasiadou et al., "Itch assessment with visual analogue scale and numerical rating scale: determination of minimal clinically important difference in chronic itch," Acta Dermato-Venereologica, vol. 96, no. 7, pp. 978-980, 2016.

[22] J. W. Lai, H. C. Chen, C. Y. Chou et al., "Transformation of 5$\mathrm{D}$ itch scale and numerical rating scale in chronic hemodialysis patients," BMC Nephrology, vol. 18, no. 1, pp. 56-61, 2017.

[23] A. Reich, E. Chatzigeorkidis, C. Zeidler et al., "Tailoring the cut-off values of the visual analogue scale and numeric rating scale in itch assessment," Acta Dermato-Venereologica, vol. 97, no. 6, pp. 759-760, 2017.

[24] S. Elman, L. S. Hynan, V. Gabriel, and M. J. Mayo, “The 5-D itch scale: a new measure of pruritus," The British Journal of Dermatology, vol. 162, no. 3, pp. 587-593, 2010.

[25] D. Amtmann, K. McMullen, J. Kim et al., "Psychometric properties of the modified 5-D itch scale in a burn model system sample of people with burn injury," Journal of Burn Care \& Research, vol. 38, no. 1, pp. e402-e408, 2017.

[26] Y. Ishiuji, Y. Umezawa, N. Aizawa et al., "Evaluation of the clinical characteristics of pruritus in patients with psoriasis using the Japanese version of the 5-D itch scale," Journal of Dermatological Science, vol. 86, no. 2, pp. e49-e49, 2017.

[27] M. Calcagno Lüer, C. S. Diez, E. Troncoso Olchevskaia, E. Ramirez Pittaluga, and R. Macchiavello Macho, "Spanish translation and cultural adaptation of the 5-D itch scale in burn patients," Burns, 2021.

[28] S.-Y. Chen, C.-C. Lai, H.-M. Chang, H.-C. Hsu, and H.-C. Pai, "Chinese version of psychometric evaluation of self-reflection and insight scale on Taiwanese nursing students," The Journal of Nursing Research, vol. 24, no. 4, pp. 337-346, 2016.

[29] I. U. Rehman and T. M. Khan, "Validity and reliability of the Urdu version of the 5D itching scale to assess pruritus among patients with chronic kidney disease in Pakistan," $B M C$ Nephrology, vol. 18, no. 1, pp. 302-302, 2017.

[30] S. Gilbody, D. Richards, S. Brealey, and C. Hewitt, "Screening for depression in medical settings with the Patient Health Questionnaire (PHQ): a diagnostic meta-analysis," Journal of General Internal Medicine, vol. 22, no. 11, pp. 1596-1602, 2007.

[31] C. Han, S. A. Jo, J.-H. Kwak et al., "Validation of the Patient Health Questionnaire-9 Korean version in the elderly population: the Ansan geriatric study," Comprehensive Psychiatry, vol. 49, no. 2, pp. 218-223, 2008.

[32] B. Levis, A. Benedetti, and B. D. Thombs, "Accuracy of Patient Health Questionnaire-9 (PHQ-9) for screening to detect major depression: individual participant data meta-analysis," $B M J$, vol. 365, article 11476, 2019.

[33] J. A. Hanley and B. J. McNeil, "The meaning and use of the area under a receiver operating characteristic (ROC) curve," Radiology, vol. 143, no. 1, pp. 29-36, 1982.

[34] C. He, B. Levis, K. E. Riehm et al., "The accuracy of the Patient Health Questionnaire-9 algorithm for screening to detect major depression: an individual participant data meta-analysis," Psychotherapy and Psychosomatics, vol. 89, no. 1, pp. 25-37, 2020.

[35] L. R. McKnight-Eily, C. A. Okoro, T. W. Strine et al., "Racial and ethnic disparities in the prevalence of stress and worry, mental health conditions, and increased substance use among adults during the COVID-19 pandemic - United States, April and May 2020," MMWR. Morbidity and Mortality Weekly Report, vol. 70, no. 5, pp. 162-166, 2021.

[36] J. Lee, H. Suh, H. Jung, M. Park, and J. Ahn, “Association between chronic pruritus, depression, and insomnia: A crosssectional study," JAAD International, vol. 3, pp. 54-60, 2021.

[37] M. M. Ruprecht, X. Wang, A. K. Johnson et al., "Evidence of social and structural COVID-19 disparities by sexual orientation, gender identity, and race/ethnicity in an urban environment," Journal of Urban Health, vol. 98, no. 1, pp. 27-40, 2021.

[38] H. L. Tey and G. Yosipovitch, "Itch in ethnic populations," Acta Dermato-Venereologica, vol. 90, no. 3, pp. 227-234, 2010. 